\title{
Glucose tolerance and mortality, including a substudy of tolbutamide treatment
}

\author{
W.C. Knowler ${ }^{1}$, G . Sartor ${ }^{2}$, A . M elander ${ }^{3}$, B. Scherstén ${ }^{4}$ \\ ${ }^{1}$ National Institute of Diabetes and Digestive and Kidney Diseases, Phoenix, Arizona, USA, and Department of Community \\ Health Sciences, Lund University, Lund, Sweden \\ ${ }^{2}$ Department of Internal Medicine, Central Hospital, Halmstad, Sweden \\ ${ }^{3}$ The NEPI Foundation, Medical Research Centre, Malmö University Hospital, Malmö, Sweden \\ ${ }^{4}$ Department of Community Health Sciences, Lund University, Lund, Sweden
}

Summary Mortality according to glucose tolerance was studied to determine the prognosis of impaired glucose tolerance. Among 2500 persons tested in a community screening programme in 1962-1965 and followed-up for mortality to the end of 1987, agesex-adjusted mortality rates were $37.9 \pm 1.9$, $53.6 \pm 4.2$, and $70.1 \pm 3.6$ deaths per 1000 personyears $( \pm \mathrm{SE})$ in those with normal glucose tolerance, impaired glucose tolerance, and diabetes by World Health Organization criteria at baseline. Age-sex-adjusted mortality rates due to ischaemic heart disease were $14.3 \pm 1.1,16.3 \pm 2.4$, and $25.8 \pm 2.0$ deaths per 1000 person-years, respectively. Using criteria predating those of the World Health Organization 147 men with abnormal glucose tolerance were entered into a randomized clinical trial in which 49 were treated with tolbutamide for approximately 10 years. Those treated had lower mortality rates from all causes (mortality rate ratio $=0.66,95 \%$ confidence inter$\mathrm{val}=0.39,1.10)$ and from ischaemic heart disease (mortality rate ratio $=0.42,95 \%$ confidence inter$\mathrm{val}=0.16,1.12)$ than those not receiving tolbutamide. Thus mortality rates are increased in persons with impaired glucose tolerance and diabetes, and the small clinical trial suggests that tolbutamide may be beneficial in men with abnormal glucose tolerance. [Diabetologia (1997) 40: 680-686]

Keywords Impaired glucose tolerance, mortality, tolbutamide, ischaemic heart disease, clinical trial.
Mortality rates are increased by non-insulin-dependent diabetes mellitus, but in many studies lesser degrees of hyperglycaemia, now referred to as impaired glucose tolerance, are also associated with higher mortality rates, especially from cardiovascular disease or sudden death [1-7]. In these studies mortality rates were 1.2 to 3 times as high in those with impaired glucose tolerance as in those with normal glucose tolerance at baseline. In two other studies, mortality rates were not significantly higher in impaired glucose tolerance $[8,9]$, although the confidence intervals were so wide that the results were compatible with a large (2-3-fold) increase in risk. These studies

Received: 22 November 1996 and in revised form: 4 March 1997

Corresponding author: Dr. W.C. Knowler, National Institute of Diabetes and Digestive and Kidney Diseases, 1550 E. Indian School Road, Phoenix, AZ 85014, USA have used various definitions of abnormal blood or plasma glucose responses during an oral glucose tolerance test, referred to as 'borderline' diabetes or impaired glucose tolerance. Criteria for impaired glucose tolerance have varied considerably, even in the two currently most widely used classification systems, those of the United States National Diabetes Data Group [10] and the World Health Organization [11]. The preponderance of evidence is that mortality rates are elevated in impaired glucose tolerance. It is not known, however, whether treatment can lower the mortality rates in persons with impaired glucose tolerance, however defined.

\section{Subjects and methods}

G lucose tolerance. Glucose tolerance tests were conducted in 2500 subjects with glycosuria discovered in a health survey in Malmöhus County, Sweden, from 1962 to 1966, as described 
Table 1. Age-sex specific frequency of impaired glucose tolerance and diabetes by World Health Organization criteria in persons with glycosuria, at the baseline examinations, 19621966

\begin{tabular}{llccc}
\hline Sex & $\begin{array}{l}\text { Age } \\
\text { (years) }\end{array}$ & $\begin{array}{l}\text { No. of } \\
\text { Subjects }^{\mathrm{a}}\end{array}$ & $\begin{array}{l}\text { Impaired } \\
\text { glucose } \\
\text { tolerance }\end{array}$ & $\begin{array}{l}\text { Diabetes } \\
(\%)\end{array}$ \\
\hline Male & $15-24$ & 80 & 6.3 & 2.5 \\
& $25-34$ & 141 & 2.1 & 2.1 \\
& $35-44$ & 231 & 6.9 & 10.8 \\
& $45-54$ & 388 & 8.0 & 18.0 \\
& $55-64$ & 456 & 12.1 & 28.3 \\
& $65-74$ & 368 & 13.3 & 35.6 \\
& $75-84$ & 128 & 24.2 & 32.0 \\
Female & $85-87$ & 5 & 20.0 & 40.0 \\
& $15-24$ & 59 & 0.0 & 3.4 \\
& $25-34$ & 53 & 3.8 & 3.8 \\
& $35-44$ & 89 & 9.0 & 19.1 \\
& $45-54$ & 129 & 6.2 & 27.9 \\
& $55-64$ & 203 & 9.4 & 54.7 \\
& $65-74$ & 128 & 15.6 & 62.5 \\
Total & $75-84$ & 42 & 11.9 & 76.2 \\
\hline
\end{tabular}

${ }^{a}$ Fifteen of these subjects could not be classified because one of the required glucose concentrations was missing

by Brandt et al. [12]. Urine was collected $2-4 \mathrm{~h}$ after a meal and tested with Clinistix (Ames Co.). Those with glycosuria were asked to return for a fasting oral glucose tolerance test, using $30 \mathrm{~g}$ glucose per $\mathrm{m}^{2}$ body surface area [12].

For comparability with other studies, the diagnostic values recommended by the World Health Organization[11] were used, even though the World Health Organization specifies a load of $75 \mathrm{~g}$ regardless of body size. By World Health Organization criteria, diabetes is diagnosed if the fasting capillary blood glucose is $6.7 \mathrm{mmol} / \mathrm{l}$ or more or the 2 -h glucose is $1.1 \mathrm{mmol} / \mathrm{l}$ or more; and impaired glucose tolerance is diagnosed if the fasting glucose is under $6.7 \mathrm{mmol} / \mathrm{l}$ and the 2 -h glucose is $7.8 \mathrm{mmol} / \mathrm{l}$ or more and under $11.1 \mathrm{mmol} / \mathrm{l}$. Other results are considered normal (by World Health Organization criteria) in this paper. The results of the glucose tolerance tests of 2500 persons with glycosuria on screening are shown in Table 1 .

Eligibility for the treatment study was based on glucose tolerance classified by the criteria herein referred to as the 'Brandt criteria' [12], which preceded the World Organization criteria [11]. Diabetes was diagnosed if the $1-\mathrm{h}$ post-load capillary blood glucose was $11.1 \mathrm{mmol} / \mathrm{l}$ or more, the 2 -h glucose was $8.6 \mathrm{mmol} / \mathrm{l}$ or more, and the $3-\mathrm{h}$ glucose was $5.8 \mathrm{mmol} / \mathrm{l}$ or more. If these criteria were not met, but at least one of the following values was found -1 -h glucose $8.9 \mathrm{mmol} / \mathrm{l}$ or more, 2 -h glucose $6.7 \mathrm{mmol} / \mathrm{l}$ or more, or 3-h glucose $4.7 \mathrm{mmol} / \mathrm{l}$ - the diagnosis was 'diabetes?', herein referred to as abnormal glucose tolerance by the Brandt criteria.

Treatment study. A randomized clinical trial was conducted in 147 men with abnormal glucose tolerance using the Brandt criteria to test whether treatment would prevent the development of diabetes [13]. After referral from a study physician, a subject was assigned to one of three groups by the pharmacist by a rotational scheme unknown to the physicians. This assignment is considered 'randomization' in this paper. Subjects in all three groups were told they were at high risk of diabetes and given the same advice, namely to increase physical activity, limit intake of carbohydrates and lipids, and, if overweight, limit total energy intake. This advice was given by a physician at the time of randomization and was repeated once each year.

In addition to diet and exercise advice, those in one group ('tolbutamide group') were treated with tolbutamide $0.5 \mathrm{~g}, 3$ times per day, those in the second group (placebo group) with placebo 3 times per day, and those in the third group (no-tablet group) were given neither tolbutamide nor placebo. All the men were randomized between 28 May 1963 and 29 November 1965 . The identity of the tolbutamide or placebo tablets was masked from subjects and investigators until 1972, when the investigators performed an interim analysis because of safety concerns raised by the University Group Diabetes Program [14]. The randomly assigned treatments were continued until 1975 , when the surviving subjects in whom diabetes had not already been diagnosed were retested for diabetes. At that time treatment was discontinued and further systematic contact with the subjects was not maintained by the investigators, but mortality rates have been determined. Results are presented according to the random treatment assignment regardless of subsequent compliance.

M ortality. Because death statistics of all residents of Sweden are reported by the national personal identification number, the date and cause of death could be obtained for each deceased subject from Statistics Sweden, Stockholm. Records for all deaths occurring from the beginning of the study through the end of 1987 were obtained, with approval from the ethics committee, Lund University.

The underlying and contributory causes of death were coded centrally according to the Swedish adaptations of the International Classification of Diseases. Version 7 was used in 1962-1968, version 8 in 1969-1986, and version 9 in 1987. The underlying cause was classified as ischaemic heart disease if the code was 420 in version 7 or $410-414$ in versions 8 or 9. In the computation of mortality rates due to ischaemic heart disease, classification was made from the underlying cause. Contributory causes are also coded on Swedish death certificates, and data were analysed by counting a death as due to ischaemic heart disease if either the underlying or a contributory cause was ischaemic heart disease. These results were similar to using the underlying cause only and are not shown.

Age-sex-specific mortality rates, from all causes and from ischaemic heart disease, were computed according to glucose tolerance for all subjects excluding the $147 \mathrm{men}$ in the treatment study. Rates were computed as deaths per 1000 personyears of observation in each decade of age, by methods described in detail previously [15]. As there were few deaths or person-years of observation below the age of 25 years, followup and deaths were counted only beyond this age. Age- adjusted and age-sex-adjusted mortality rates and their standard errors were computed by the direct method [15], using as a reference population the age and sex distribution of all subjects participating in the population screening.

Mortality rates were also computed with the proportional hazards model [16], which allows for adjustment for age and other factors as continuous variables. The estimated hazard rates were converted to cumulative mortality rates for presentation [16]. The mortality rates from ischaemic heart disease were computed by coding deaths from other causes as censored observations. Thus, these cause-specific cumulative mortality rates estimate the proportion of subjects who would have died from ischaemic heart disease had other causes of death not intervened. 
Table 2. Age-sex specific all-cause mortality rates (deaths/1000 person-years) in subjects with glycosuria on screening, by glucose tolerance according to World Health Organization criteria at baseline

\begin{tabular}{|c|c|c|c|c|c|c|c|c|c|}
\hline \multirow{2}{*}{$\begin{array}{l}\text { Age } \\
\text { (years) }\end{array}$} & \multicolumn{3}{|c|}{ Normal glucose tolerance } & \multicolumn{3}{|c|}{ Impaired glucose tolerance } & \multicolumn{3}{|c|}{ Diabetes } \\
\hline & $\mathrm{P}-\mathrm{yr}^{\mathrm{a}}$ & Deaths & Rate & $\mathrm{P}-\mathrm{yr}^{\mathrm{a}}$ & Deaths & Rate & $\mathrm{P}-y r^{\mathrm{a}}$ & Deaths & Rate \\
\hline \multicolumn{10}{|l|}{ M en } \\
\hline $45-54$ & 3680 & 22 & 6.0 & 250 & 4 & 16.0 & 523 & 4 & 7.7 \\
\hline $55-64$ & 4762 & 59 & 12.4 & 478 & 10 & 20.9 & 1354 & 38 & 28.1 \\
\hline $65-74$ & 4533 & 141 & 31.1 & 666 & 40 & 60.1 & 1842 & 116 & 63.0 \\
\hline \multicolumn{10}{|l|}{ Women } \\
\hline $25-34$ & 801 & 1 & 1.3 & 8 & 0 & 0.0 & 33 & 0 & 0.0 \\
\hline $35-44$ & 1268 & 2 & 1.6 & 47 & 0 & 0.0 & 108 & 0 & 0.0 \\
\hline $45-54$ & 1494 & 4 & 2.7 & 126 & 0 & 0.0 & 366 & 4 & 10.9 \\
\hline $55-64$ & 1637 & 12 & 7.3 & 222 & 5 & 22.6 & 965 & 17 & 17.6 \\
\hline $65-74$ & 1350 & 21 & 15.6 & 310 & 10 & 32.3 & 1359 & 75 & 54.2 \\
\hline \multicolumn{3}{|c|}{ Age-sex-adjusted rate } & 37.9 & & & 53.6 & & & 70.1 \\
\hline \multicolumn{3}{|c|}{$\begin{array}{l}95 \% \text { confidence interval } \\
{ }^{a} \text { Person-years of follow-up }\end{array}$} & $34.2-4$ & & & $45.4-6$ & & & $63.1-77.2$ \\
\hline
\end{tabular}

\section{Results}

Mortality by glucose tolerance. Age-sex-specific allcause mortality rates by glucose tolerance according the World Health Organization criteria are shown in Table 2, excluding the 147 men in the treatment study. In general, mortality rates were lowest in those with normal glucose tolerance, intermediate in those with impaired glucose tolerance, and highest in those with diabetes. In pairwise comparisons between the groups, adjusted for age and sex, the differences were highly statistically significant (impaired compared with normal glucose tolerance, age-sex adjusted mortality rate ratio $=1.41,95 \%$ confidence interval $=1.18,1.68$; diabetes compared with normal, age-sex-adjusted mortality rate ratio $=2.01,95 \%$ confidence interval $=1.78,2.26$ ). Age- sex-adjusted all-cause mortality rates were $37.9 \pm 1.9,53.6 \pm 4.2$, and $70.1 \pm 3.6$ deaths per 1000 person-years $( \pm$ SE) in those with normal glucose tolerance, impaired glucose tolerance, and diabetes, respectively, by World Health Organization criteria at baseline (Table 2). Age-sex-adjusted mortality rates due to ischaemic heart disease were $14.3 \pm 1.1, \quad 16.3 \pm 2.4$, and $25.8 \pm 2.0$ deaths per 1000 person-years, respectively. The age-adjusted rates by sex are shown in Figure 1 for all causes and from ischaemic heart disease according to World Health Organization criteria for glucose tolerance.

Effect of tolbutamide on mortality in the treatment study. At baseline, the distributions of glucose tolerance, age, blood glucose concentrations, and blood pressure in the three treatment groups are shown in
Table 3. There were no important or statistically significant differences, as expected from the randomization.

Follow-up was computed for each man from the date of his randomization (1963-1965) until death or 31 December 1987, the closing date of this analysis. Because all subjects were randomized by the end of 1965, the survivors had at least 22 years of follow-up until 31 December 1987. By the end of follow-up, 75 subjects had died, including 27 deaths due to ischaemic heart disease.

Estimates of 20-year cumulative mortality rates are shown in Table 4 for men in each of the treatment groups and in all other men $(n=682)$ with abnormal glucose tolerance by the Brandt criteria. Although the men in the three treatment groups were very similar at baseline (Table 3), they were younger and differed in other respects from the other 682 men with abnormal glucose tolerance. Thus age-adjusted mortality rates were compared in a proportional hazards model containing variables for age and each of the treatment groups. Although there were no significant differences between the four groups in this model, rates were slightly higher in those treated with placebo and lower in those treated with tolbutamide. The untreated group had similar all-cause mortality rates to those treated with diet but no tablets. The age-adjusted mortality rate for all treated men as a group was nearly identical to that of untreated men (mortality rate ratio $=1.02,95 \%$ confidence inter$\mathrm{val}=0.79,1.30)$. Similarly, the age-adjusted rate of deaths from ischaemic heart disease was nearly the same in the treated and untreated men (mortality rate ratio $=1.09,95 \%$ confidence interval $=0.72$, 1.66). The analyses in Table 4 were also adjusted for 


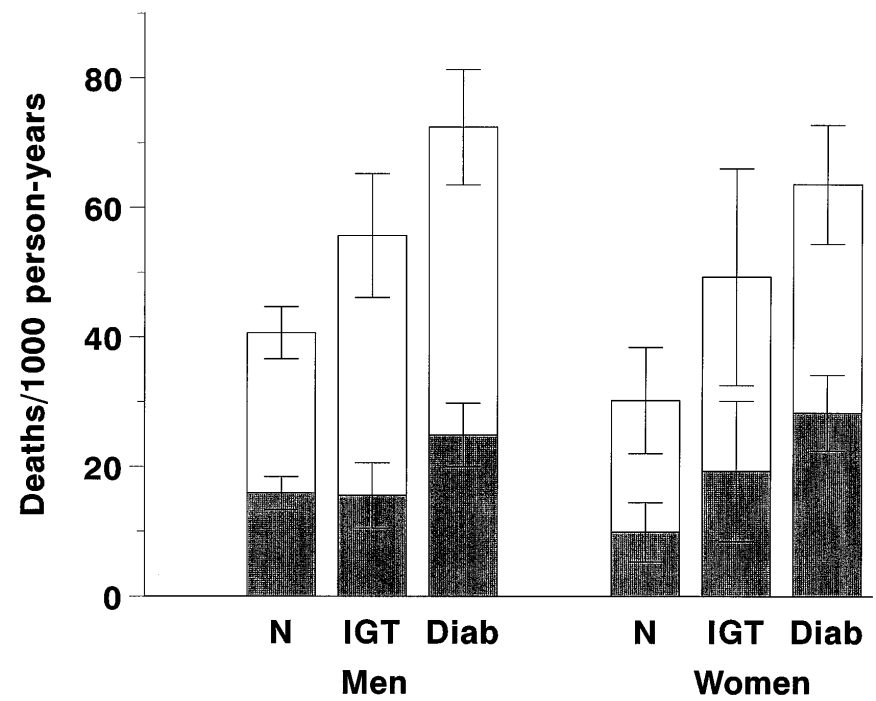

Fig. 1. Age-adjusted mortality rates (deaths/1000 person-years through 1987) with $95 \%$ confidence intervals due to all causes (full height of bar) and due to ischaemic heart disease (shaded part of bar) according to glucose tolerance at baseline by World Health Organization criteria: N, normal glucose tolerance; IGT, impaired glucose tolerance; Diab, diabetes

baseline blood pressure and blood glucose; the results were similar and are not shown.

Because follow-up results were similar in the placebo and no-tablet groups (especially for ischaemic heart disease), these two groups are combined in subsequent results as a 'no tolbutamide' group. The cumulative mortality rates by follow-up time are shown in Figure 2. Rates of deaths due to all causes are shown on the left and those due to ischaemic heart disease on the right, and are age-adjusted to a value of 50 years at baseline with the proportional hazards model. The 20- year cumulative mortality rates in Figure 2 do not agree exactly with Table 4, because they were computed from a model using only the 147 treated men (who had a different age distribution than all the subjects shown in Table 4). Because the mean ages did not differ between the treatment groups, the crude (i.e. not age-adjusted) mortality rates were very similar to the age-adjusted rates shown in Figure 2. The treatment differences were similar in analyses that also adjusted for other baseline variables, such as blood glucose or blood pressure (not shown), since these variables were also very similar between the treatment groups (Table 3). The cumulative mortality rates from all causes and from ischaemic heart disease were lower in the men treated with tolbutamide. In the proportional hazards analysis controlled for age, the mortality rate ratios of those treated with tolbutamide (compared with those not) were 0.66 (95\% confidence interval $=0.39,1.10$ ) for all causes of death and $0.42(95 \%$ confidence interval $=0.16,1.12)$ for ischaemic heart disease.
Table 3. Baseline characteristics of men with impaired glucose tolerance by Brandt [12] criteria ${ }^{\mathrm{a}}$ in the randomized treatment study, 1963-1965

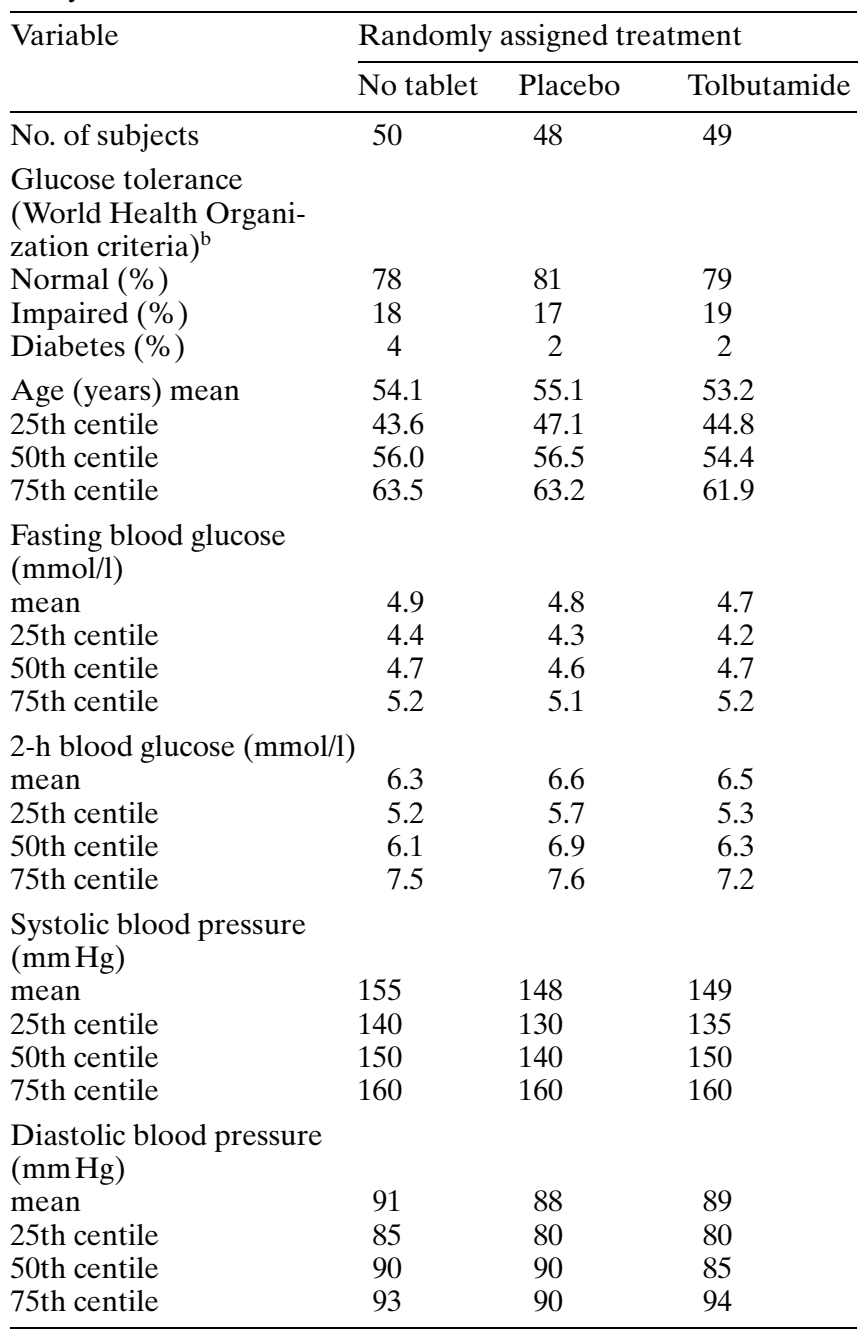

${ }^{a}$ In reviewing the study records of each subject, it was discovered that 6 ( 3 in the tolbutamide group, 1 in the placebo group, and 2 in the no-tablet group) had not met the Brandt criteria at baseline, but were nevertheless considered by their physicians to have impaired glucose tolerance and thus randomized in the study

${ }^{b}$ World Health Organization classification could not be made for one subject treated with tolbutamide because the fasting blood glucose concentration was missing. Judged by the 2-h blood glucose only, he would have had normal glucose tolerance by World Health Organization criteria

Neither the distribution of glucose tolerance categories nor the means of any of these continuous variables differed significantly between the three treatment groups

\section{Discussion}

Mortality rates are reported in a long-term follow-up of 2500 persons given oral glucose tolerance tests after having glycosuria on a screening examination. In agreement with previous studies [1-7], age-sex adjusted mortality rates were twice as high in diabetic subjects as in those with normal glucose tolerance, 
Table 4. Twenty-year cumulative mortality rates in men with abnormal glucose tolerance by Brandt criteria and in the three treatment groups

\begin{tabular}{lcll}
\hline Group & $\begin{array}{l}\text { Number } \\
\text { of men }\end{array}$ & $\begin{array}{l}\text { 20-year cumulative mortality } \\
\text { rate }(\%)\end{array}$ \\
\cline { 2 - 4 } & & All causes & $\begin{array}{l}\text { Ischaemic } \\
\text { heart disease }\end{array}$ \\
\hline Untreated & 682 & 30 & 11 \\
Diet, no tablets & 50 & 31 & 15 \\
Diet + placebo & 48 & 37 & 15 \\
Diet + tolbutamide & 49 & 24 & 7 \\
\hline
\end{tabular}

Estimated from a proportional hazards model derived from all four groups of men, with variables for age and each treatment group, adjusted to a baseline age of 50 years. There were no significant differences between the four groups in mortality rates due to all causes or ischaemic heart disease
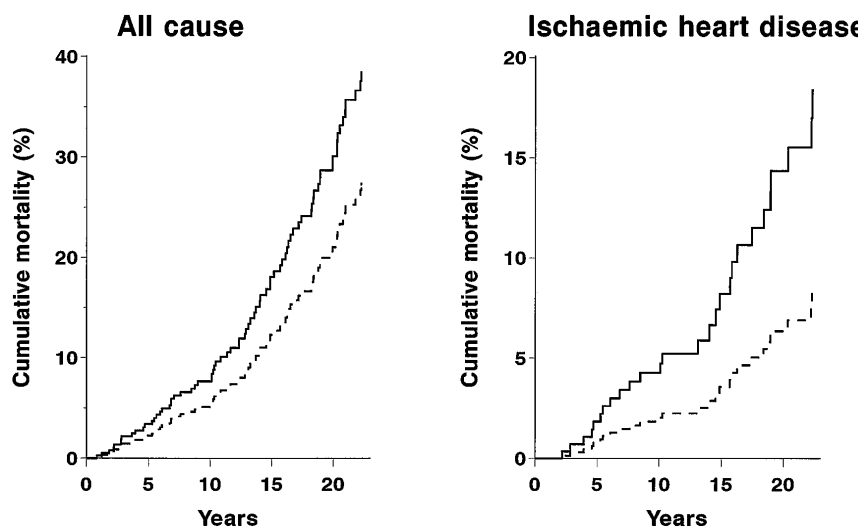

Fig. 2. Cumulative incidence (\%) of death due to all causes (left) and due to ischaemic heart disease (right) in 147 men according to randomization for treatment of impaired glucose tolerance with (- - - - - -) or without (_ Mortality rates are age-adjusted with the proportional hazards model and evaluated at an age of 50 years at baseline. The cumulative mortality rates from all causes (mortality rate ratio $=0.66,95 \%$ confidence interval $=0.39,1.10)$, and from ischaemic heart disease (mortality rate ratio $=0.42,95 \%$ confidence interval $=0.16,1.12$ ) were lower in the men treated with tolbutamide

and mortality rates in subjects with impaired glucose tolerance were intermediate. In the Rancho Bernardo, California, study, ischaemic heart disease death rates in men were linearly and positively associated with fasting plasma glucose [17]. In the present study, mortality rates due to all causes and to ischaemic heart disease were related to hyperglycaemia as classified by the World Health Organization criteria (Fig. 1) or by the Brandt criteria or other categories of fasting or post-load blood glucose (not shown). Thus hyperglycaemia predicts mortality rates regardless of the particular scheme used to classify hyperglycaemia $[1-7,17]$.

A subset of 147 men with abnormal glucose tolerance by Brandt criteria were randomly assigned to treatment with or without tolbutamide. As a whole, they had similar mortality rates to the other, untreated, men with abnormal glucose tolerance (Table 4). Because these groups differed in ways other than their treatment, comparisons between the treated and untreated men must be interpreted cautiously and it is difficult to judge whether the treatments as a whole affected mortality rates. No differences in baseline characteristics were apparent, however, among the 147 treated men, allowing for a comparison of their mortality rates by type of treatment. There were no significant effects of the treatment on mortality rates when the 10-year treatment was completed [13]. The slight differences apparent at 10 years increased, however, when follow-up was extended to 22 years or more (Fig. 2). The majority of these men would not have impaired glucose tolerance if their tests were interpreted by World Health Organization criteria (Table 3), although this interpretation is not precisely correct because the oral glucose challenge was not the same as that recommended by the World Health Organization. Thus generalization of these results to those who would currently be classified as having impaired glucose tolerance or diabetes is uncertain.

The treatment study was stopped in 1975, after which time the men received medical care from a large number of practitioners. Thus it was not feasible to ascertain what treatment they received after 1975, nor which subjects developed diabetes. Because Swedish mortality statistics are collected centrally, however, it was possible to determine the mortality rates and causes of death for an additional 12 years. Reliance was made on the centrally reported statistics without verification from individual medical records, which were not readily available. Such statistics have been shown to be especially accurate for the coding of cardiovascular diseases in Sweden [18, 19].

Some of the problems of interpreting the 10-year diabetes incidence results [13] remain unresolved: a) The effect of dietary treatment cannot be evaluated because there was no randomized control group; all of the men in the treatment group were given the same dietary advice. b) Assessment of the tolbutamide effect is difficult because many subjects discontinued treatment before the treatment study was concluded in 1975. Analysis by randomization to treat with tolbutamide showed no significant effect, but comparison of those continuing to take tolbutamide with those assigned to no drug or placebo, and to those who discontinued taking tolbutamide, suggested that the drug prevented diabetes [13].

The important conclusion from the present paper is that, whether or not diabetes was prevented or delayed, randomization to tolbutamide treatment may have reduced all-cause mortality rates by $34 \%$ (i.e. mortality rate ratio $=0.66,95 \%$ confidence inter$\mathrm{val}=0.39,1.10)$, and mortality rates from ischaemic 
heart disease by $58 \%$ (mortality rate ratio $=0.42$, $95 \%$ confidence interval $=0.16,1.12$ ). The uncertainty of this conclusion comes from the small sample size and resulting wide confidence intervals for the treatment effects. These estimates were not substantially affected by further adjustment for other cardiovascular risk factors measured at baseline, although confounding by unmeasured risk factors such as smoking and serum cholesterol remains a possibility.

At no time was there any suggestion of a harmful effect of tolbutamide, either in terms of incidence of diabetes, total mortality, or death from ischaemic heart disease (Fig. 2), in agreement with the clinical trial of tolbutamide treatment of impaired glucose tolerance in Bedford, England [20]. This conclusion differs from that of the University Group Diabetes Program randomized clinical trial of treatment of diabetes [14]. The University Group Diabetes Program was terminated early because of a higher rate of death due to cardiovascular disease in subjects assigned to tolbutamide than in those assigned to insulin or placebo. As no significant difference in allcause mortality rates was reported in that study, it is unclear whether the drug treatment actually increased mortality rates or resulted in a difference in the attributed causes of death. A major problem with the interpretation of these mortality results is that the study was terminated before planned based on cause-specific, but not total, mortality rates. The Malmöhus study group was not faced with this problem, as there were no important mortality rate differences at the time the study was discontinued for other reasons. It was only with continued mortality follow-up, long after the trial had ended, that mortality rate differences appeared. The results are still uncertain, however, because the confidence intervals for the effects are very wide due to the small size of the treatment study (only 49 men were assigned to tolbutamide treatment). Thus future clinical trials in this area must be much larger if mortality rates are to be studied, and subjects should be followed long after the randomized clinical trial is completed.

In conclusion, metabolic abnormalities manifest as overt diabetes or as abnormalities on the oral glucose tolerance test (impaired glucose tolerance or diabetes) predict increased age- sex specific mortality rates, both total and from heart disease. These findings confirm that glucose intolerance is associated with cardiovascular disease, but they do not indicate whether glucose intolerance causes the cardiovascular disease or simply results from the same risk factors [3]. Firm conclusions about treatment effects cannot be drawn from this small study because the apparent reduction in mortality rates, while large, was not statistically significant. Yet, the possible benefit of treatment with tolbutamide suggests that glucose intolerance is at least in part causally related to ischaemic heart disease and, furthermore, that attempts at correcting it are worthwhile.

A cknowledgements. We thank the participants in the screening and treatment studies for their cooperation, Drs. Sven Carlström (deceased), Åke Nordén, and Gunnar Persson for conducting the treatment study, Per Nyberg for research assistance, and Karin Svenninger and Åke Isacsson for administrative support. The mortality analysis was supported in part by the Medical Research Council, Stockholm, Sweden.

\section{References}

1. Jarrett RJ, McCartney P, Keen H (1982) The Bedford Survey: Ten year mortality rates in newly diagnosed diabetics, borderline diabetics and normoglycaemic controls and risk indices for coronary heart disease in borderline diabetics. Diabetologia 22: 79-84

2. Fuller JH, Shipley MJ, Rose G, Jarrett RJ, Keen H (1983) Mortality from coronary heart disease and stroke in relation to degree of glycaemia: the Whitehall Study. BMJ 287: $867-870$

3. Jarrett RJ, Shipley MJ (1988) Type 2 (non-insulin- dependent) diabetes mellitus and cardiovascular disease - putative association via common antecedents; further evidence from the Whitehall Study. Diabetologia 31: 737-740

4. Smith GD, Egger M, Shipley MH, Marmot MG (1992) Post-challenge glucose concentration, impaired glucose tolerance, diabetes, and cancer mortality in men. Am J Epidemiology 136: 1110-1114

5. Balkau B, Eschwège E, Papoz L, et al. (1993) Risk factors for early death in non-insulin dependent diabetes and men with known glucose tolerance status. BMJ 307: 295- 299

6. Sigurdsson E, Sigfusson N, Agnarsson U, Sigvaldason H, Thorgeirsson G (1995) Long-term prognosis of different forms of coronary heart disease: the Reykjavik Study. Int J Epidemiology 24: 58-68

7. Curb JD, Rodriquez BL, Burchfiel CM, Abbott RD, Chiu D, Yano K (1995) Sudden death, impaired glucose tolerance, and diabetes in Japanese American men. Circulation 91: 2591-2595

8. Stengård JH, Tuomilehto J, Pekkanen J, et al. (1992) Diabetes mellitus, impaired glucose tolerance and mortality among elderly men: The Finnish cohorts of the Seven Countries Study. Diabetologia 35: 760-765

9. Tuomilehto J, Schranz A, Aldana D, Pitkäniemi J (1994) The effect of diabetes and impaired glucose tolerance on mortality in Malta. Diabet Med 11: 170-176

10. National Diabetes Data Group (1979) Classification and diagnosis of diabetes mellitus and other categories of glucose intolerance. Diabetes 28: 1039-1057

11. Report of a WHO Study Group (1985) Diabetes Mellitus. World Health Organization Tech Rep Ser 727: 9-17

12. Brandt L, Nordén A, Scherstén B, Tryding N (1964) A diabetes detection campaign in southern Sweden. Results of 69,000 examinations. Acta Med Scand 176: 555561

13. Sartor G, Scherstén B, Carlström S, Melander A, Norden $\AA$, Persson G (1980) Ten-year follow-up of subjects with impaired glucose tolerance: prevention of diabetes by tolbutamide and diet regulation. Diabetes 29: 41-49

14. University Group Diabetes Program (1970) A study of the effects of hypoglycemic agents on vascular complications in patients with adult-onset diabetes. II. Mortality results. Diabetes 19 [Suppl 2]:789-830 
15. Knowler WC, Bennett PH, Hamman RF, Miller M (1978) Diabetes incidence and prevalence in Pima Indians: a 19fold greater incidence than in Rochester, Minnesota. Am J Epidemiol 108: 497-505

16. Kalbfleisch JD, Prentice RL (1980) The statistical analysis of failure time data. John Wiley \& Sons, New York

17. Scheidt-Nave C, Barrett-Connor E, Wingard DL, Cohn BA, Edelstein SL (1991) Sex differences in fasting glycemia as a risk factor for ischaemic heart disease death. Am J Epidemiol 133: 565-576
18. Britton M (1974) Diagnostic errors discovered at autopsy. Acta Med Scand 196: 203-210

19. de Faire U, Friberg L, Lorich U, Lundman T (1976) A validation of cause-of-death certification in 1156 deaths. Acta Med Scand 200: 223-228

20. Keen H, Jarrett RH, Fuller JH (1974) Tolbutamide and arterial disease in borderline diabetics. In: Malaisse WJ, Pirart J (eds) Diabetes: Proceedings of the Eighth Congress of the International Diabetes Federation. Excerpta Medica, Amsterdam, pp 588-602 